\title{
On the $I(G)$-adic Topology of the Burnside Ring of Compact Lie Groups
}

By

\author{
Norihiko MINAMI*
}

\section{Introduction}

Let $G$ be a compact Lie group and let $A(G)$ denote tom Dieck's Burnside ring [2], [3, 5.5]. As a set, $A(G)$ consists of equivalence classes of compact $G$-ENR's, and as a module, $A(G)$ is a free abelian group with basis $\{G / H\}$, where $H$ runs over $\Phi(G)$ : the set of conjugacy classes $(H)$ such that $N H / H$ is finite. Here $(H)$ denotes the conjugacy class of $H$ in $G$ and $N H$ the normalizer of $H$.

In $[3,5.12]$ tom Dieck defined homomorphisms between $A(G)$ and $A(H)$;

$$
\begin{aligned}
& \operatorname{Res}_{H}^{G}: A(G) \longrightarrow A(H) \\
& I n d_{H}^{G}: A(H) \longrightarrow A(G)
\end{aligned}
$$

where $\operatorname{Res}_{H}^{G}$ is $r_{H}^{G}$ and $I n d_{H}^{G}$ is $e_{H}^{G}$ in tom Dieck's notation. Now let $e$ be the trivial subgroup of $G$. Then

$$
\operatorname{Res}_{e}^{G}: A(G) \longrightarrow A(e) \cong Z \text { (=the ring of integers) }
$$

defines the augmentation ideal $I(G)$ as its kernel.

In this paper we shall study the $I(G)$-adic topology of $A(G)$. For this purpose we make use of the particular subgroups of $G$. Let $T$ be a maximal torus of $G$. Since the Weyl group $N T / T$ is finite, we choose a $p$-Sylow subgroup $F_{p}$ of $N T / T$ for each prime $p$ dividing the order of $N T / T$. We set $N_{p}=\pi^{-1}\left(F_{p}\right)$ where $\pi: N T \rightarrow N T / T$ denotes the canonical projection.

In Section 1 we show that the $I\left(N_{p}\right)$-adic topology is the same as

Communicated by N. Shimada, December 22, 1982.

* Research Institute for Mathematical Sciences, Kyoto University, Kyoto 606, Japan.

Current address : Department of Mathematics, Faculty of Science, Hiroshima University, Hiroshima 730, Japan. 
its $p$-adic topology. In Section 2 we show that

$$
\operatorname{Ind}_{N_{p}}^{G}: A\left(N_{p}\right) \longrightarrow A(G)
$$

is continuous. In Section 3 we explicitly determine the structure of $A(G)^{\wedge}$ : the $I(G)$-adic completion of $A(G)$.

Recently G. Carlsson solved the Segal conjecture for a finite group whose weak form asserts that $A(G)^{\wedge}$ is canonically isomorphic to $\pi_{s}^{0}\left(B G_{+}\right)$: the 0 -th stable cohomotopy of the classifying space of $G[1]$. Therefore one naturally wonders if the Segal conjecture could be generalized to the case of compact Lie groups. We show however in Corollary 3.6 that if the action of the Weyl group on the maximal torus is non-trivial then the generalization of the Segal conjecture does not hold. Therefore the $I(G)$-adic topology is not appropriate when one considers such a problem.

Quite recently, G. Nishida developed a good device to handle $\pi_{s}^{*}\left(B G_{+}\right)$[7]. Along his line one may solve the Segal conjecture affirmatively, in the case of a central extention of a finite group by a torus (this is exactly the case when the action of the Weyl group on the maximal torus is trivial!). Note that this is the same as saying that $A(G)$ is Noetherian [2, Proposition 10] [3, 5.10.8].

The author wishes to express his gratitude to Professor Nobuo Shimada for his heartful encouragement and he also thanks to Professors Goro Nishida and Akira Kono for many valuable and heartful suggestions.

\section{§1. $I\left(N_{p}\right)$-adic Topology of $I\left(N_{p}\right)$}

The purpose of this section is to prove the following

Proposition 1.1. If $G$ is an extension of a finite $p$-group by a torus, then the $I(G)$-adic topology of $I(G)$ is the same as its p-adic topology.

First we recall some results of tom Dieck. With a suitable topology $\Phi(G)$ becomes a countable, totally disconnected, compact Hausdorff space $[3,5.6 .1]$. Let $C(\Phi(G), Z)$ denote the ring of continuous functions of $\Phi(G)$ to $Z$. For the conjugacy class $(H)$ of a closed subgroup $H$ of $G$ there is a ring homomorphism

$$
\phi_{H}: A(G) \longrightarrow Z
$$


which assigns an integer $\chi\left(X^{H}\right)$ to a compact $G$-ENR $X$ where $X^{H}$ denotes the $H$-stationary subspace of $X$ and $\chi$ the Euler number. Then we have the well defined ring homomorphism

$$
\dot{\phi}: A(G) \longrightarrow C(\phi(G), Z)
$$

such that

$$
\phi(X):(H) \longmapsto \phi_{H}(X) .
$$

tom Dieck [3, 5.8.5] characterized this correspondence, generalizing the result of A. Dress [5, 1.3] as follows.

Theorem A. The ring homomorphism

$$
\phi: A(G) \longrightarrow C(\Phi(G), Z)
$$

is an embedding and $z \in C(\Phi(G), Z)$ is contained in $\phi A(G)$ if and only if for all $(H) \in \Phi(G)$

$$
\sum_{(K)} n(H, K) z(K) \equiv 0 \bmod |N H / H|
$$

where the summation is taken over the $N H$-conjugacy classes $(K)$ such that $K \triangleright H$ and $K / H$ is cyclic, and $n(H, K)$ are the integers defined in $[3,5.8 .4]$.

Moreover tom Dieck provides us the finiteness property of the Burnside ring [3, 5.9.9], though it is not Noetherian in general [2, Proposition 10], [3, 5.10.8].

Theorem B. There exists an integer $b$ such that for each closed subgroup $H$ of $G$ the index $\left|(N H / H):(N H / H)_{0}\right|$ is less than b. Here $(\mathrm{NH} / \mathrm{H})_{0}$ denotes the identity component of $\mathrm{NH} / \mathrm{H}$.

Lemma 1.2. Let $G$ be as in Proposition 1.1. Then for any closed subgroup $H$ we have

$$
\phi_{H} \equiv \chi \bmod p
$$

as homomorphisms of $A(G)$ to $Z$.

Proof. Let $T$ be a maximal torus of $G$. For a compact $G$-ENR $X$, we have

$$
\phi_{H}(X)=\chi\left(X^{H}\right)=\chi\left(\left(X^{H \cap T}\right)^{H / H \cap T}\right) \equiv \chi\left(X^{H \cap T}\right) \bmod p
$$


and

$$
\chi\left(X^{H \cap T}\right)=\chi\left(\left(X^{H \cap T}\right)^{T / H \cap T}\right)=\chi\left(X^{T}\right)=\chi(X)
$$

where the latter half follows from the fact that $T / H \cap T$ and $T$ are tori.

Proposition 1.3. Let $G$ be as in Proposition 1.1. Then for any closed subgroup $H$ of $G$ the index $\left|N H / H:(N H / H)_{0}\right|$ is a power of $p$.

Proof. Let $1 \longrightarrow T \longrightarrow G \stackrel{\pi}{\longrightarrow} F \longrightarrow 1$ be the exact sequence. Then we find immediately that

$$
\begin{aligned}
& \mid N H / H:(N H / H)_{0}|=| N H \cap T / H \cap T:(N H \cap T / H \cap T)_{0} \mid \\
& \times|\pi(N H)| /|\pi(H)| .
\end{aligned}
$$

Since $N H \cap T / H \cap T=(T / H \cap T)^{\pi(H)}$, it suffices to show that the index $\left|(T / H \cap T)^{\pi(H)}:\left((T / H \cap T)^{\pi(H)}\right)_{0}\right|$ is a power of $p$.

Now recall the canonical isomorphism [8, 1.5.6]

$$
H^{0}(\pi(H), T / H \cap T) \cong(T / H \cap T)^{\pi(H)} /\left(\sum_{\sigma \in \pi(H)} \sigma\right) T / H \cap T .
$$

Note that the left hand side cohomology is the Tate cohomology [8, 1.4.7]. Since $\left(\sum_{\sigma \in \pi(H)} \sigma\right) T / H \cap T$ is connected and $H^{0}(\pi(H), T / H \cap T)$ is $|\pi(H)|$-torsion $[8,3.1 .6]$, we have

$$
\left(\sum_{\sigma \in \pi(H)} \sigma\right) T / H \cap T=\left((T / H \cap T)^{\pi(H)}\right)_{0}
$$

and consequentely $(T / H \cap T)^{\pi(H)} /\left((T / H \cap T)^{\pi(H)}\right)_{0}$ is a finite $p$-group as desired.

Proof of Proposition 1.1. We have to show that for each natural number $m$ there are natural numbers $n_{1}, n_{2}$ such that

(i ) $\quad p^{n_{1}} I(G) \subset I(G)^{m}$,

(ii) $\quad I(G)^{n_{2}} \subset p^{m} I(G)$.

For the relation (i) we show $|N T / T| \cdot I(G)^{n} \subset I(G)^{n+1}$. Let $x$ be an element of $I(G)^{n}$. Then we have

$$
\begin{aligned}
|N T / T| x & =(|N T / T|-G / T) \cdot x+G / T \cdot x \\
& =(|N T / T|-G / T) \cdot x+\operatorname{Ind}_{T}^{G} \operatorname{Res}_{T}^{G} x \\
& =(|N T / T|-G / T) \cdot x \in I(G) \cdot I(G)^{n}=I(G)^{n+1} .
\end{aligned}
$$


We turn to the relation (ii). For any compact $G$-ENR $X$, we have

$$
\phi_{H}(X-\chi(X))=\left(\phi_{H}-\chi\right)(X) \equiv 0 \bmod p
$$

by Lemma 1.2. So we find

$$
\phi I(G) \subset p C(\Phi(G), Z) \cap\{f \in C(\Phi(G), Z) \mid f(T)=0\} .
$$

As $\dot{\phi}$ is a ring homomorphism,

$$
\phi\left(I(G)^{n}\right) \subset p^{n} C(\Phi(G), Z) \cap\{f \in C(\Phi(G), Z) \mid f(T)=0\} .
$$

On the other hand, by Theorem A, Theorem B and Proposition 1.3 there is a natural number $s$ such that

$$
p^{s} C(\Phi(G), Z) \cap\{f \in C(\Phi(G), Z) \mid f(T)=0\} \subset \phi I(G) .
$$

Consequently we have

$$
\begin{aligned}
\phi\left(I(G)^{m+s}\right) & \subset p^{m+s} C(\Phi(G), Z) \cap\{f \in C(\Phi(G), Z) \mid f(T)=0\} \\
& \subset p^{m} \phi I(G)=\phi\left(p^{m} I(G)\right) .
\end{aligned}
$$

Since $\phi$ is injective, we have proved the relation (ii).

\section{§2. Continuity of $\operatorname{Ind}_{N_{p}}^{G}$}

Let $G$ be a compact Lie group and let $H$ and $K$ be arbitrary closed subgroups of $G$. Consider $G / H \times G / K$ as a compact $G$-ENR. Then we have the decomposition

$$
G / H \times G / K=\cup(G / H \times G / K)_{\left(K \cap H^{g}\right)}
$$

into the subspaces of an orbit type. Let $(G / H \times G / K)_{\left(K \cap H^{g}\right), b}$ be the inverse image in $(G / H \times G / K)_{\left(K \cap H^{g}\right)}$ of a connected component of $(G / H \times G / K)_{\left(K \cap H^{g}\right)} / G$. So the index $b$ distinguishes the components. Then we have a decomposition

$$
G / H \times G / K=\sum n_{\left(K \cap H^{g}\right), b} G / K \cap H^{g}
$$

in $A(G)$.

Now again tom Dieck provides us the double coset formula for the Burnside ring [3, 5.12.13].

Theorem C. With the above notations, we have

$$
\operatorname{Res}_{K}^{G} \operatorname{Ind} d_{H}^{G}=\sum n_{\left(K \cap H^{g}\right) . b} \operatorname{Ind}_{K \cap H^{g}}^{K} \operatorname{Res}_{K \cap H^{g}}^{H^{g}} C_{g}
$$


where $C_{g}$ is the conjugation by $g$.

We apply the double coset formula to the case of $H=N_{p}$ and $K=N_{q}$ where $p$ and $q$ are distinct.

Proposition 2.1. If the $N_{q}$-conjugacy class of $N_{q} \cap N_{p}^{g}$ belongs to $\Phi\left(N_{q}\right)$, then $N_{q} \cap N_{p}^{g}=T^{g}=T$.

Proof. First we show that $N_{q} \cap N_{p}^{g}$ is always abelian. Let $\pi_{1}$ : $N_{q} \longrightarrow F_{q}$ and $\pi_{2}: N_{p}^{g} \longrightarrow F_{p}^{g}$ denote the canonical projections. Then from the exact sequences

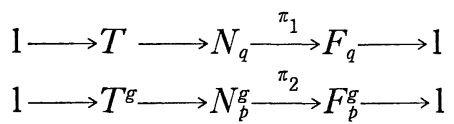

we get the following ones :

$$
\begin{aligned}
& 1 \longrightarrow T \cap N_{p}^{g} \longrightarrow N_{q} \cap N_{p}^{g} \stackrel{\pi_{1}}{\longrightarrow} \pi_{1}\left(N_{q} \cap N_{p}^{g}\right) \longrightarrow 1 \\
& 1 \longrightarrow T^{g} \cap N_{q} \longrightarrow N_{p}^{g} \cap N_{q} \stackrel{\pi_{2}}{\longrightarrow} \pi_{2}\left(N_{p}^{g} \cap N_{q}\right) \longrightarrow 1 .
\end{aligned}
$$

Since $\left(\left|\pi_{1}\left(N_{q} \cap N_{p}^{g}\right)\right|,\left|\pi_{2}\left(N_{p}^{g} \cap N_{q}\right)\right|\right)=1$, we easily find that the following sequences are also exact.

$$
\begin{aligned}
& 1 \longrightarrow T \cap T^{g} \longrightarrow N_{q} \cap N_{p}^{g} \stackrel{\pi_{1} \times \pi_{2}}{\longrightarrow} \pi_{1}\left(N_{q} \cap N_{p}^{g}\right) \times \pi_{2}\left(N_{q} \cap N_{p}^{g}\right) \longrightarrow 1 \\
& 1 \longrightarrow T \cap T^{g} \longrightarrow N_{q} \cap T^{g} \stackrel{\pi_{1}}{\longrightarrow} \pi_{1}\left(N_{q} \cap N_{p}^{g}\right) \longrightarrow 1 \\
& 1 \longrightarrow T \cap T^{g} \longrightarrow T \cap N_{p}^{g} \stackrel{\pi_{2}}{\longrightarrow} \pi_{2}\left(N_{q} \cap N_{p}^{g}\right) \longrightarrow 1
\end{aligned}
$$

The extensions (ii) and (iii) are central because $N_{q} \cap T^{g}$ and $T \cap N_{p}^{g}$ are abelian. Therefore the extension (i) is also central.

Let $\alpha$ be the element in

$$
H^{2}\left(\pi_{1}\left(N_{q} \cap N_{p}^{g}\right) \times \pi_{2}\left(N_{q} \cap N_{p}^{g}\right), T \cap T^{g}\right)
$$

which corresponds to the central extension (i). Let $i_{j}(j=1,2)$ denote the canonical inclusion

$$
i_{j}: \pi_{j}\left(N_{q} \cap N_{p}^{g}\right) \longrightarrow \pi_{1}\left(N_{q} \cap N_{p}^{g}\right) \times \pi_{2}\left(N_{q} \cap N_{p}^{g}\right) \quad(j=1,2)
$$

and let $p_{j}(j=1,2)$ denote the canonical projection

$$
p_{j}: \pi_{1}\left(N_{q} \cap N_{p}^{g}\right) \times \pi_{2}\left(N_{q} \cap N_{p}^{g}\right) \longrightarrow \pi_{j}\left(N_{q} \cap N_{p}^{g}\right) \quad(j=1,2) .
$$

Then we find immediately that

$$
\left(i_{1}^{*} \times i_{2}^{*}\right) \alpha=\left(i_{1}^{*} \times i_{2}^{*}\right)\left(p_{1}^{*} i_{1}^{*} \alpha+p_{2}^{*} i_{2}^{*} \alpha\right)
$$


holds in

$$
H^{2}\left(\pi_{1}\left(N_{q} \cap N_{p}^{g}\right), T \cap T^{g}\right) \oplus H^{2}\left(\pi_{2}\left(N_{q} \cap N_{p}^{g}\right), T \cap T^{g}\right) .
$$

As

$$
\begin{aligned}
i_{1}^{*} \times i_{2}^{*}: & H^{2}\left(\pi_{1}\left(N_{q} \cap N_{p}^{g}\right) \times \pi_{2}\left(N_{q} \cap N_{p}^{g}\right), T \cap T^{g}\right) \\
& \longrightarrow H^{2}\left(\pi_{1}\left(N_{q} \cap N_{p}^{g}\right), T \cap T^{g}\right) \oplus H^{2}\left(\pi_{2}\left(N_{q} \cap N_{p}^{g}\right), T \cap T^{g}\right)
\end{aligned}
$$

is an isomorphism, we get

$$
\alpha=p_{1}^{*} i_{1}^{*} \alpha+p_{2}^{*} i_{2}^{*} \alpha \text {. }
$$

Now $p_{1}^{*} i_{1}^{*} \alpha$ corresponds to the extension

$$
1 \longrightarrow T \cap T^{g} \longrightarrow N_{q} \cap T^{g} \times \pi_{2}\left(N_{q} \cap N_{p}^{g}\right) \longrightarrow \pi_{1}\left(N_{q} \cap N_{p}^{g}\right) \times \pi_{2}\left(N_{q} \cap N_{p}^{g}\right) \longrightarrow 1
$$

and $p_{2}^{*} i_{2}^{*} \alpha$ corresponds to the extension

$1 \longrightarrow T \cap T^{g} \longrightarrow \pi_{1}\left(N_{q} \cap N_{p}^{g}\right) \times T \cap N_{p}^{g} \longrightarrow \pi_{1}\left(N_{q} \cap N_{p}^{g}\right) \times \pi_{2}\left(N_{q} \cap N_{p}^{g}\right) \longrightarrow 1$.

Therefore $p_{1}^{*} i_{1}^{*} \alpha$ and $p_{2}^{*} i_{2}^{*} \alpha$ correspond to the extensions which yield abelian groups by the exact sequences (ii) and (iii). Hence so does $\alpha$ by the definition of the Baer multiplication [8, 5.1]. Therefore $N_{q} \cap N_{p}^{g}$ is abelian.

Now we proceed further. If $\pi_{2}\left(N_{q} \cap N_{p}^{g}\right)$ is trivial, $N_{q} \cap N_{p}^{g}$ is contained in $T^{g}$. Then the assertion follows immediately. Therefore we suppose that $\pi_{2}\left(N_{q} \cap N_{p}^{g}\right)$ is non-trivial.

Since $N_{q} \cap N_{p}^{g}$ is abelian, we can set

$$
N_{q} \cap N_{p}^{g}=C \oplus F_{p}^{\prime}
$$

where $G$ is a closed subgroup of $N_{q} \cap N_{p}^{g}$ such that

$$
\left(p,\left|C: C_{0}\right|\right)=1
$$

and $F_{p}^{\prime}$ is a non trivial finite $p$-subgroup of $N_{q} \cap N_{p}^{g}$.

Then we find that $N_{q} \cap N_{p}^{g} / C$ is a non-trivial $p$-subgroup of $N_{N_{q}} G / C$. By Proposition 1.3 we have $(C) \notin \Phi\left(N_{q}\right)$. It follows that $(T / C \cap T)^{\pi_{1}\left(N_{q} \cap N_{p}^{g}\right)}$ is infinite by an easy argument [4], [3, 5.10.6].

Now consider the following fiber bundle of which the projection is $\pi_{1}\left(N_{q} \cap N_{p}^{g}\right)$ - equivariant.

$$
N_{q} \cap N_{p}^{g} \cap T / C \cap T \longrightarrow T / C \cap T \longrightarrow T / N_{q} \cap N_{p}^{g} \cap T
$$

Since the fibre $N_{q} \cap N_{p}^{g} \cap T / C \cap T \cong F_{p}^{\prime}$ is finite,

$$
\left(T / N_{q} \cap N_{p}^{g} \cap T\right)^{\pi_{1}\left(N_{q} \cap N_{p}^{g}\right)}
$$

is infinite too. Therefore we get 


$$
\left(N_{q} \cap N_{p}^{g}\right) \notin \Phi\left(N_{q}\right)
$$

and this proves Proposition 2.1.

As a corollary of the double coset formula we have the following.

Corollary 2.2. $\operatorname{Res}_{N_{q}}^{G} \operatorname{Ind} d_{N_{p}}^{G}: I\left(N_{p}\right) \longrightarrow I\left(N_{q}\right)$ is a zero map when $p$ and $q$ are distinct.

Proposition 2.3. $\operatorname{Ind}_{N_{p}}^{G}\left(\left|F_{p}\right|^{n} I\left(N_{p}\right)\right) \subset I(G)^{n} I n d_{N_{p}}^{G} I\left(N_{p}\right)$.

Proof. We shall prove by induction on $n$. The case $n=0$ is trivial. Suppose that the case $n-1$ is proved. Let $y$ be an element of $\operatorname{Ind}_{N_{p}}^{G}\left(\left|F_{p}\right|^{n} I\left(N_{p}\right)\right)$. We can set $y=\left|F_{p}\right| x$ where $x$ belongs to $I(G)^{n-1} I n d_{N_{p}}^{G}\left(I\left(N_{p}\right)\right)$ by the inductive step.

Since the G. C. D. of $\left\{\left|(N T / T) / F_{q}\right|\right\}_{q \neq p}$ is $\left|F_{p}\right|$, there are integers $\left\{n_{q}\right\}_{q \neq p}$ such that

$$
\left|F_{p}\right|=\sum_{q \neq p} n_{q}\left|(N T / T) / F_{q}\right| \text {. }
$$

Hence it suffices to show that $\left|(N T / T) / F_{q}\right| x \quad(q \neq p)$ belongs to $I(G)^{n} \operatorname{In} d_{N_{p}}^{G} I\left(N_{p}\right)$.

Now we have the equality

$$
\begin{aligned}
\left|(N T / T) / F_{q}\right| x & =\left(\chi(G / N T) \chi\left(N T / N_{q}\right)-G / N_{q}\right) x+\operatorname{Ind}_{N_{q}}^{G} \operatorname{Res}_{N_{q}}^{G} x \\
& =\left(\chi\left(G / N_{q}\right)-G / N_{q}\right) x+\operatorname{Ind}_{N_{q}}^{G} \operatorname{Res}_{N_{q}}^{G} x .
\end{aligned}
$$

Since $\operatorname{Res}_{N_{q}}^{G} x=0$ by Corollary 2.2, we get

$$
\begin{aligned}
\left|(N T / T) / F_{q}\right| x & =\left(\chi\left(G / N_{q}\right)-G / N_{q}\right) x \\
& \in I(G) I(G)^{n-1} \operatorname{Ind}_{N_{p}}^{G} I\left(N_{p}\right)=I(G)^{n} \operatorname{Ind}_{N_{p}}^{G} I\left(N_{p}\right)
\end{aligned}
$$

as required.

Theorem 2.4. Ind ${ }_{N_{p}}^{G}: A\left(N_{p}\right) \longrightarrow A(G)$ is continuous. Hence it induces $\hat{\operatorname{Ind}} d_{N_{p}}^{G}: A\left(N_{p}\right)^{\wedge} \longrightarrow A(G)^{\wedge}$.

Proof. This easily follows from Proposition 1.1 and Proposition 2.3.

Remark. $\operatorname{Res}_{H}^{G}: A(G) \longrightarrow A(H)$ is always continuous because $\operatorname{Res}_{H}^{G}$ 
is a ring homomorphism which preserves the augmentations. Therefore it induces $\hat{R} e s_{H}^{G}: A(G)^{\wedge} \longrightarrow A(H)^{\wedge}$.

\section{§3. The Structure of $\boldsymbol{A}(\boldsymbol{G})^{\wedge}$}

Since the G. C. D. of $\left\{\left|(N T / T) / F_{p}\right|\right\}_{p}$ is 1 , there are integers $\left\{m_{p}\right\}_{p}$ such that $\sum_{p} m_{p}\left|(N T / T) / F_{p}\right|=1$.

Now define the homomorphisms

$$
\begin{aligned}
& \text { Ind }: \bigoplus_{p} A\left(N_{p}\right)^{\wedge} \longrightarrow A(G)^{\wedge} \\
& \text { Res }: A(G)^{\wedge} \longrightarrow \bigoplus_{p} A\left(N_{p}\right)^{\wedge}
\end{aligned}
$$

by

$$
\begin{array}{ll}
\hat{I} n d\left(\underset{p}{\bigoplus_{p}} x_{p}\right)=\sum_{p} m_{p} \hat{I} n d_{N_{p}}^{G} x_{p}, & x_{p} \in A\left(N_{p}\right)^{\wedge} \\
\hat{R} e s(y)=\bigoplus_{p} \hat{R} e s_{N_{p}}^{G} y, & y \in A(G)^{\wedge}
\end{array}
$$

Lemma 3.1. (i) $\operatorname{Ind}(1)=\sum_{p} m_{p} G / N_{p}$ is a unit in $A(G)^{\wedge}$. (ii) $\hat{I n d}(z \cdot \hat{R} e s(w))=\hat{\operatorname{Ind}}(z) \cdot w, z \in \bigoplus_{p} A\left(N_{p}\right)^{\wedge}, \quad w \in A(G)^{\wedge}$.

Proof. (i) easily follows from

$$
\chi\left(G / N_{p}\right)=\chi(G / N T) \chi\left(N T / N_{p}\right)=\left|(N T / T) / F_{p}\right| .
$$

We turn to the case (ii). It suffices to show that

$$
\hat{I}_{n d_{N_{p}}^{G}}\left(x \cdot \hat{\operatorname{R}} e s_{N_{p}}^{G} y\right)=\hat{\operatorname{In}} d_{N_{p}}^{G}(x) \cdot y, x \in A\left(N_{p}\right)^{\wedge}, y \in A(G)^{\wedge} .
$$

Consider the commutative diagram

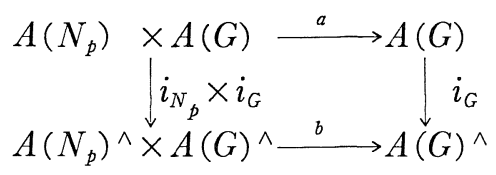

where $i_{N_{p}}$ and $i_{G}$ are obvious homomorphisms; while $a$ and $b$ are defined as follows ;

$$
\begin{aligned}
& a(s, t)=0\left(=\operatorname{Ind}_{N_{p}}^{G}\left(s \cdot \operatorname{Res}_{N_{p}}^{G}(t)\right)-\operatorname{In} d_{N_{p}}^{G}(s) \cdot t\right) \\
& b(x, y)=\hat{I} n d_{N_{p}}^{G}\left(x \cdot \hat{\operatorname{R}} s_{N_{p}}^{G}(y)\right)-\hat{\operatorname{In}} d_{N_{p}}^{G}(x) \cdot y
\end{aligned}
$$

for every $s \in A\left(N_{p}\right), t \in A(G), x \in A\left(N_{p}\right)^{\wedge}, y \in A(G)^{\wedge}$.

Regard the modules of the above diagram as topological spaces of 
$I\left(N_{p}\right)$-adic on $A\left(N_{p}\right)$ and $A\left(N_{p}\right)^{\wedge}$, and of $I(G)$-adic on $A(G)$ and $A(G)^{\wedge}$. Then the above diagram is that of topological spaces and continuous maps. Since $\left(i_{N_{p}} \times i_{G}\right)\left(A\left(N_{p}\right) \times A(G)\right)$ is dense in $A\left(N_{p}\right)^{\wedge}$ $\times A(G)^{\wedge}$ and $A(G)^{\wedge}$ is Hausdorff, we have

$$
\begin{aligned}
b\left(A\left(N_{p}\right)^{\wedge} \times A(G)^{\wedge}\right) & =b \overline{\left(\left(i_{N_{p}} \times i_{G}\right)\left(A\left(N_{p}\right) \times A(G)\right)\right.} \\
& =\overline{b\left(\left(i_{N_{p}} \times i_{G}\right)\left(A\left(N_{p}\right) \times A(G)\right)\right.} \\
& =\overline{i_{G} a\left(A\left(N_{p}\right) \times A(G)\right)}=\overline{\{0\}}=\{0\} .
\end{aligned}
$$

So the result follows.

As an immediate consequence, we have

Corollary 3.2. Ind $\hat{R} e s$ is an endomorphism of $A(G)^{\wedge}$.

Remark. This fact essentially shows that one can reduce the Segal conjecture to the case of an extension of a finite $p$-group by a torus (cf. [6]). Though such a result is not so essential as we shall see later (Corollary 3.6.), our argument yields a very short proof of Theorem A of [6] because we can use $[5,1.14]$ to prove Theorem 2.4 if $G$ is a finite group.

Lemma 3.3. $\operatorname{Res}_{N_{p}}^{G} \operatorname{Ind}_{N_{p}}^{G} I\left(N_{p}\right): \operatorname{Ind}_{N_{p}}^{G} I\left(N_{p}\right) \longrightarrow I\left(N_{p}\right)$ and

are injective.

$$
\hat{\operatorname{R}} e s_{N_{p}}^{G} \mid \hat{\operatorname{In}} d_{N_{p}}^{G} I\left(N_{p}\right)^{\wedge}: \hat{\operatorname{In}} d_{N_{p}}^{G} I\left(N_{p}\right)^{\wedge} \longrightarrow I\left(N_{p}\right)^{\wedge}
$$

Proof. We first prove that $\operatorname{Res}_{N_{p}}^{G} \mid \operatorname{Ind}_{N_{p}}^{G} I\left(N_{p}\right)$ is injective.

Note that $\operatorname{In} d_{N_{p}}^{G} I\left(N_{p}\right)$ is a free abelian group with basis $\{G / H-$ $\left.\chi\left(N_{p} / H\right) G / N_{p}\right\}$ where $H$ runs over the representatives of $(H) \in \Phi(G)$ such that one of its conjugates is a proper subgroup of $N_{p}$. Therefore for any non-zero element $z$ of $\operatorname{Ind}_{N_{p}}^{G} I\left(N_{p}\right)$ we can write it down as

$$
z=\sum_{\lambda} n_{\lambda} G / H_{\lambda}, \quad H_{\lambda} \subset N_{p}, \quad\left(H_{\lambda}\right) \in \Phi(G)
$$

where the summation is a finite sum such that one of $n_{\lambda}$ is non zero. Now choose a maximal subgroup $H_{\lambda}$ such that $n_{\lambda}$ is non zero. 
Then we find

$$
\chi_{H_{\lambda}}(z)=n_{\lambda}\left|N H_{\lambda} / H_{\lambda}\right| \neq 0 \text {. }
$$

This implies that $\operatorname{Res}_{N_{p}}^{G}(z)$ is also non-zero.

We now turn to the case of $\hat{R} e s_{N_{p}}^{G} \mid \hat{I} n d_{N_{p}}^{G} I\left(N_{p}\right)^{\wedge}$. Since $\hat{R} e s$ is injective, we need only to show that $\hat{R} e s_{N_{q}}^{G} \hat{I} \eta d_{N_{p}}^{G}$ is a zero map when $p$ and $q$ are distinct. But this is evident.

By the second part of Lemma 3.3, we have a topological module isomorphism

$$
\begin{aligned}
I(G)^{\wedge} & \cong \hat{I} n d\left(\bigoplus_{p} I\left(N_{p}\right)^{\wedge}\right) \cong \underset{p}{\bigoplus_{p}} \hat{R} e s_{N_{p}}^{G} \hat{I}_{n} d_{N_{p}}^{G} I\left(N_{p}\right)^{\wedge} \\
& \cong \bigoplus_{p} \hat{I}_{n} d_{N_{p}}^{G} I\left(N_{p}\right)^{\wedge}
\end{aligned}
$$

where the topology of $\hat{R} e s_{N_{p}}^{G} \hat{I} n d_{N_{p}}^{G} I\left(N_{p}\right)^{\wedge}$ is the subspace topology of $I\left(N_{p}\right)^{\wedge}$; since the former is a direct summand of the latter, it is $p$ adic topology. Similarly the topology of $\operatorname{In}_{N_{p}}^{G} I\left(N_{p}\right)^{\wedge}$ is $p$-adic.

Now for any module $M$, we denote its $p$-adic completion by $M_{p}^{\wedge}$.

Proposition 3.4. $\hat{I}_{n d_{N_{p}}^{G}} I\left(N_{p}\right)^{\wedge} \cong\left(\hat{\operatorname{In}} d_{N_{p}}^{G} I\left(N_{p}\right)\right)_{p}^{\wedge}$.

Proof. Since $\operatorname{In} d_{N_{p}}^{G} I\left(N_{p}\right)$ is a free abelian group and $\hat{I} n d_{N_{p}}^{G} \hat{R} e s_{N_{p}}^{G}$ is an automorphism of $\hat{I}_{n} d_{N_{p}}^{G} I\left(N_{p}\right)^{\wedge}$, each sequence of the following commutative diagram splits.

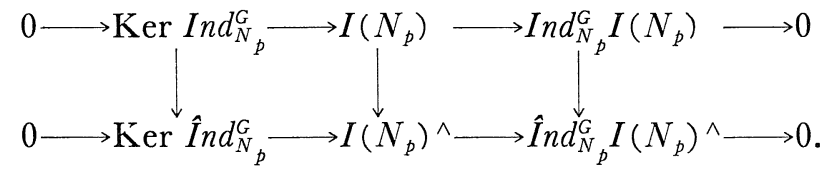

Applying the $p$-adic completion functor to this, one gets the following one.

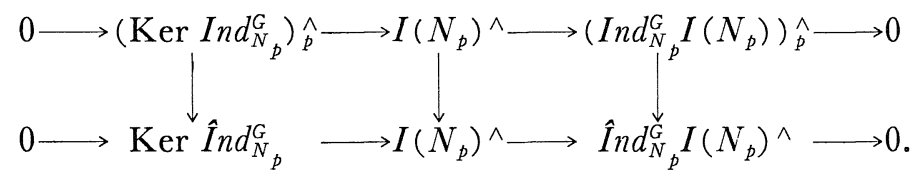

Note that $\operatorname{Ind}_{N_{p}}^{G} \operatorname{Res}_{N_{p}}^{G}: \operatorname{In} d_{N_{p}}^{G} I\left(N_{p}\right) \longrightarrow \operatorname{Ind}_{N_{p}}^{G} I\left(N_{p}\right)$ is a multiplication by $G / N_{p}$ which is an automorphism of $\left(\operatorname{Ind}_{N_{p}}^{G} I\left(N_{p}\right)\right) \hat{p}$ by Lemma 1.2. Therefore the above diagram is a map of exact sequences which 
commutes with the splittings $\left(\operatorname{Res}_{N_{p}}^{G}\right) \hat{p}$ and $\hat{R} e s_{N_{p}}^{G}$. This implies that

$$
\hat{\operatorname{In}} d_{N_{p}}^{G} I\left(N_{p}\right)^{\wedge} \cong\left(\operatorname{Ind} d_{N_{p}}^{G} I\left(N_{p}\right)\right) \hat{p} \text {. }
$$

Now we have arrived at our main theorem.

Theorem 3.5. As topological modules we have

$$
\begin{aligned}
& A(G)^{\wedge} \cong Z \bigoplus_{p} \underset{p}{\bigoplus_{p}}\left(\operatorname{Ind}_{N_{p}}^{G} I\left(N_{p}\right)\right) \hat{p}, \\
& I(G)^{\wedge} \cong \bigoplus_{p}\left(\operatorname{Ind} d_{N_{p}}^{G} I\left(N_{p}\right)\right) \hat{p} .
\end{aligned}
$$

And $\operatorname{Ind}_{N_{p}}^{G} I\left(N_{p}\right)$ is a free abelian group with basis $\left\{G / H-\chi\left(N_{p} / H\right) G / N_{p}\right\}$ where $H$ runs over the representatives of $(H) \in \phi(G)$ such that one of its conjugates is a proper subgroup of $N_{p}$. Moreover $\operatorname{Ind}_{N_{p}}^{G} I\left(N_{p}\right)$ is finitely generated if and only if the action of $F_{p}$ on $T$ is trivial.

Proof. We have only to prove the last part. But this is exactly the same as Proposition 5.10.8 of [3].

As an application of our main theorem, we shall show that the augmentation ideal-adic topology is not adequate when one considers the Segal conjecture for compact Lie groups.

Corollary 3.6. If the action of $N T / T$ on $T$ is non-trivial, then as modules $A(G)^{\wedge}\left(=Z \oplus I(G)^{\wedge}\right)$ and $\underset{\leftarrow}{\lim } \pi_{s}^{0}\left(B G_{+}^{n}\right) \quad\left(=Z \oplus \underset{\leftarrow}{\lim } \pi_{s}^{0}\left(B G^{n}\right)\right)$ are different where $X^{n}$ denotes the n-skeleton of a $C W$ complex $X$.

Proof. If the action of $N T / T$ on $T$ is non-trivial, then for some $p$ the action of $F_{p}$ on $T$ is also non-trivial. In this situation, $I(G)^{\wedge} /$ $p I(G)^{\wedge}$ is an infinite countable dimensional $Z / p$-vector space by Theorem 3.5. We show that

$$
\lim _{n} \pi_{s}^{0}\left(B G^{n}\right) / p \underset{\leftarrow}{\lim _{n}} \pi_{s}^{0}\left(B G^{n}\right)
$$

cannot be such a $Z / p$-vector space.

Let $A_{n}$ denote $\underset{m>n}{\cap} \operatorname{Im}\left(\pi_{s}^{0}\left(B G^{m}\right) \longrightarrow \pi_{s}^{0}\left(B G^{n}\right)\right)$. Consider the following exact sequence of inverse systems. 


$$
0 \longrightarrow\left\{\operatorname{Ker}\left(A_{n} \stackrel{\cdot p}{\longrightarrow} A_{n}\right)\right\} \longrightarrow\left\{A_{n}\right\} \stackrel{\circ p}{\longrightarrow}\left\{A_{n}\right\} \longrightarrow\left\{A_{n} / p A_{n}\right\} \longrightarrow 0 .
$$

Since $\left\{A_{n}\right\}$ is an inverse system of finite groups, all the inverse systems are those of finite groups. Therefore the sequence

$$
0 \longrightarrow \lim _{n} \operatorname{Ker}\left(A_{n} \stackrel{\cdot p}{\longrightarrow} A_{n}\right) \longrightarrow \underset{n}{\longrightarrow} \lim A_{n} \stackrel{\cdot p}{\longrightarrow} \lim _{n} A_{n} \longrightarrow \lim _{n} A_{n} / p A_{n} \longrightarrow 0
$$

is exact.

As $\left\{A_{n} / p A_{n}\right\}$ is a surjective inverse system of finite dimensional $Z / p$-vector spaces, we have two cases :

(i) There is a natural number $N$ such that

$$
\underset{n}{\lim } \operatorname{dim}_{Z / p} A_{n} / p A_{n}=N \text {. }
$$

(ii) $\underset{n}{\lim _{\longrightarrow}} \operatorname{dim}_{Z / p} A_{n} / p A_{n}=\infty$.

In the case of (i), $\overleftarrow{n}_{n}^{\lim } A_{n} / p A_{n}=(Z / p)^{N}$. In the case of (ii), $\stackrel{\lim }{n}_{n} A_{n} / p A_{n}$ $=\prod_{i}^{\infty}(Z / p)_{i}$ which is a non countable dimensional $Z / p$-vector space.

In any case, it can not be an infinite countable dimensional $Z / p$ vector space. Since we have

$$
\lim _{\longleftarrow} A_{n} / p A_{n}=\lim _{n} \pi_{s}^{0}\left(B G^{n}\right) / p \lim _{n} \pi_{s}^{0}\left(B G^{n}\right),
$$

the proof is now finished.

Remark. (i) Under the assumption of Corollary 3.6, we easily see that $A(G)^{\wedge}$ and $\pi_{s}^{0}\left(B G_{+}\right)$are also different to each other as topological spaces. In fact $I(G)^{\wedge}$ is not compact and $\pi_{s}^{0}(B G)$ is compact.

(ii) In many cases, the assumption of Corollary 3.6 is satisfied. For example, every non abelian compact connected Lie group does.

\section{References}

[1] Carlsson, G., Equivariant stable homotopy and Segal's Burnside ring conjecture, preprint.

[2] tom Dieck, T., The Burnside ring of a compact Lie group I, Math. Ann. 215 (1975), 235-250.

[3] Transformation groups and representation theory, Lecture Notes in Math., 766, Springer-Verlag,

[4] Gordon, R. A., The Burnside ring of a cyclic extention of a torus, Math. Z., 153 (1977), 149-153.

[5] Laitinen, E., On the Burnside ring and stable cohomotopy of a finite group, Math. 
Scand., 44 (1979), 37-72.

[6] May, J. P. and McClure, J. E., A reduction of the Segal conjecture, Canadian Mathematical Society Conference Proceding, Volume 2, Part 2 (1982), 209-222.

[ 7 ] Nishida, G., On the $S^{1}$-Segal conjecture, Publ. RIMS, Kyoto Univ., 19 (1983), 1153-1162.

[8] Weiss, E., Cohomology of Groups, Academic Press, 1969. 